\title{
Synthesis and antimicrobial screening of 5-arylidene-2-imino-4-thiazolidinones
}

\author{
Ameya A. Chavan and Nandini R. Pai* \\ Department of Organic Chemistry, D. G. Ruparel College, Senapati Bapat Marg, Mahim, \\ Mumbai-400 016, India \\ E-mail: nandini_pai@hotmail.com or ameyaachavan@gmail.com
}

\begin{abstract}
The condensation of 2-aminobenzothiazole-6-carboxylic acid (1) with chloroacetyl chloride in refluxing chloroform in the presence of anhydrous $\mathrm{K}_{2} \mathrm{CO}_{3}$ gives 2-(2chloroacetylamino)benzothiazole-6-carboxylic acid (2). Compound (2) on treatment with KSCN in refluxing acetone yields 2-(2-imino-4-oxo-thiazolidin-3-yl)benzothiazole-6-carboxylic acid (3). Compound (3) on condensation with various aromatic aldehydes affords a series of 2-[5(arylidene)-2-imino-4-oxo-thiazolidin-3-yl]benzothiazole-6-carboxylic acid (4a-h). The synthesized compounds (4a-h) are screened for their antibacterial as well as antifungal activity. All the tested compounds show slight to moderate activity against the selected microorganisms.
\end{abstract}

Keywords: Thiazolidin-4-one, 2-aminobenzothiazole, antifungal activity

\section{Introduction}

Heterocycles bearing nitrogen, sulphur and thiazole moieties constitute the core structure of a number of biologically interesting compounds. The chemistry of thiazolidin-4-one ring systems is of considerable interest as it is a core structure in various synthetic pharmaceuticals displaying a broad spectrum of biological activities. ${ }^{1}$ Thiazolidin-4-one derivatives are known to exhibit diverse bioactivities such as antidiarrheal, ${ }^{2}$ anticonvulsant, ${ }^{3}$ antimicrobial, ${ }^{4}$ antidiabetic, ${ }^{5}$ antihistaminic, ${ }^{6}$ anticancer, ${ }^{7}$ antiHIV,${ }^{8} \mathrm{Ca}^{2+}$ channel blocker, ${ }^{9}$ PAF antagonist, ${ }^{10}$ cardioprotective, ${ }^{11}$ anti-ischemic, ${ }^{12}$ cycloxygenase inhibitory, ${ }^{13}$ anti-platelet activating factor, ${ }^{14}$ non-peptide thrombin receptor antagonist ${ }^{15}$ and tumor necrosis factor- $\alpha$ antagonist activities. ${ }^{16}$ Also 2-imino-thiazolidin-4-ones have been found to have antifungal activity. ${ }^{17-19}$ Moreover literature survey reveals that 2-aminobenzothiazoles possess antimicrobial and various other pharmacological activities like diuretic, ${ }^{20}$ anticancer, ${ }^{21}$ antiulcer, ${ }^{22}$ and antihistamine. ${ }^{23}$ Hence it is thought of interest to accommodate thiazolidin-4-one and 2-amino benzothiazole moieties in single molecular framework and screen them for their antimicrobial activity. 


\section{Results and Discussion}

2-Aminobenzothiazole-6-carboxylic acid (1) is prepared in quantitative yield according to a known method. ${ }^{24}$ This on condensation with chloroacetyl chloride in the presence of anhydrous $\mathrm{K}_{2} \mathrm{CO}_{3}$ as base and chloroform as solvent gives 2-(2-chloroacetylamino)benzothiazole-6carboxylic acid (2). Compound (2) on reaction with $\mathrm{KSCN}$ in refluxing acetone yields 2-(2imino-4-oxo-thiazolidin-3-yl)benzothiazole-6-carboxylic acid (3). Condensation of compound (3) with various aromatic aldehydes affords a series of 2-[5-(arylidene)-2-imino-4-oxothiazolidin-3-yl]benzothiazole-6-carboxylic acid (4a-h). The purity of the compounds is monitored by TLC technique. The structures of the compounds are confirmed on the basis of their elemental analysis and spectral data. The physical constants and yields of the compounds (4a-h) are described in Table 1.

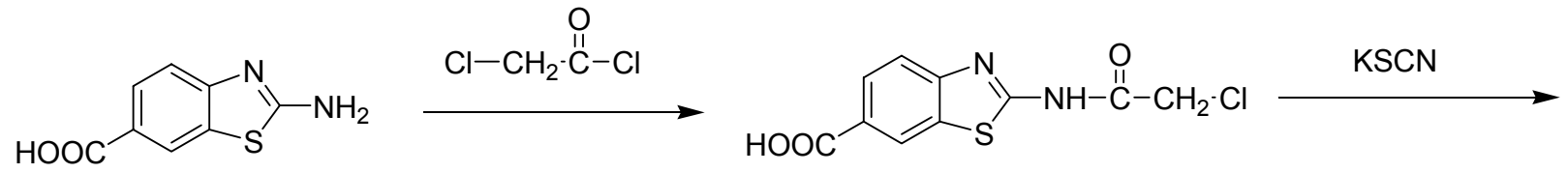

(1)

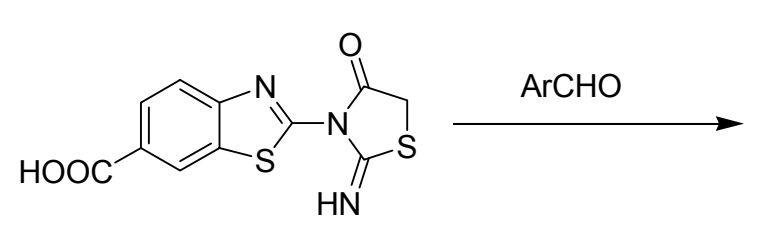

(3)
(2)<smiles>N=C1SC(=CC(Br)Br)C(=O)N1c1nc2ccc(C(=O)O)cc2s1</smiles>

$(4 a-h)$

Table 1. Structures of the compounds 4a-h, their melting points and yields of synthesis

\begin{tabular}{cccc}
\hline Compound & Substituent $\mathrm{Ar}$ & M.P. ${ }^{0} \mathrm{C}$ & Yield \% \\
\hline $\mathbf{4 a}$ & $2-\mathrm{ClC}_{6} \mathrm{H}_{4}$ & $255-257$ & 61 \\
$\mathbf{4 b}$ & $4-\mathrm{ClC}_{6} \mathrm{H}_{4}$ & $248-250$ & 59 \\
$\mathbf{4 c}$ & $4-\mathrm{OHC}_{6} \mathrm{H}_{4}$ & $244-246$ & 69 \\
$\mathbf{4 d}$ & $3-\mathrm{BrC}_{6} \mathrm{H}_{4}$ & $278-280$ & 58 \\
$\mathbf{4} \mathbf{e}$ & $4-\mathrm{OCH}_{3} \mathrm{C}_{6} \mathrm{H}_{4}$ & $251-253$ & 55 \\
$\mathbf{4 f}$ & $3-\mathrm{NO}_{2} \mathrm{C}_{6} \mathrm{H}_{4}$ & $266-268$ & 65 \\
$\mathbf{4 g}$ & $4-\mathrm{NO}_{2} \mathrm{C}_{6} \mathrm{H}_{4}$ & $273-275$ & 68 \\
$\mathbf{4 h}$ & $\mathrm{C}_{6} \mathrm{H}_{5}$ & $238-240$ & 63 \\
\hline
\end{tabular}

\section{Antibacterial activity}

Cup plate method using Hi-Media agar medium is employed to study the antibacterial activity of 4a-h against Staphylococcus aureus, Bacillus subtilis, Psuedomonas aeruginosa and Escherichia coli. ${ }^{25}$ Preparation of nutrient broth, subculture, base layer medium, agar medium and peptone 
water is done as per the standard procedure. Each test compound $(50 \mathrm{mg})$ is dissolved in $50 \mathrm{~mL}$ of Dimethyl Formamide $(1000 \mu \mathrm{g} / \mathrm{mL})$, which is used as sample solution. Sample size for all the compounds is fixed as $0.1 \mathrm{~mL}$.

The cups are made by scooping out agar medium with sterilized cork borer in a petri dish, which is previously inoculated with the microorganisms. The solution of each test compound $(0.1 \mathrm{~mL})$ is added in the cups and petri dishes are subsequently incubated at $37^{0}$ for $48 \mathrm{~h}$. Ampicillin and Streptomycin are used as reference drugs and Dimethyl Formamide as a control. Zone of inhibition produced by each compound is measured in mm, as shown in Table 2 .

All the newly synthesized compounds show antibacterial activity against $S$. aureus, B. subtilis, $P$. aeruginosa and E. coli, the data of which is presented in Table 2.

Thus a series of thiazolidin-4-ones having benzothiazole moiety into one molecular framework have been synthesized which display slight to moderate antibacterial activity.

Table 2. Antibacterial activity of the compounds $\mathbf{3}$ and $\mathbf{4 a - h}$

\begin{tabular}{ccccc}
\hline & \multicolumn{2}{c}{ Gram positive bacteria } & \multicolumn{2}{c}{ Gram negative bacteria } \\
Compound & S. aureus & B. subtilis & $P$. aeruginosa & E.coli \\
Ampicillin & +++ & ++ & ++ & +++ \\
Streptomycin & +++ & +++ & +++ & +++ \\
\hline $\mathbf{3}$ & + & - & - & + \\
$\mathbf{4 a}$ & ++ & - & + & + \\
$\mathbf{4 b}$ & ++ & - & - & + \\
$\mathbf{4 c}$ & - & + & + & ++ \\
$\mathbf{4 d}$ & + & - & + & ++ \\
$\mathbf{4 e}$ & + & + & - & ++ \\
$\mathbf{4 f}$ & - & + & ++ & ++ \\
$\mathbf{4 g}$ & + & - & + & ++ \\
$\mathbf{4 h}$ & ++ & + &
\end{tabular}

Key to symbols: Inactive $=-($ inhibition zone $<6 \mathrm{~mm}$ ); slightly active $=+$ (inhibition zone 6-9 $\mathrm{mm}$ ); moderately active $=++$ (inhibition zone $9-12 \mathrm{~mm}$ ); highly active $=+++$ (inhibition zone $>12 \mathrm{~mm})$.

\section{Antifungal activity}

The antifungal activity of compounds $\mathbf{4 a - h}$ is tested against four different fungi such as $C$. albicans, $C$. pannical, $A$. niger and $R$. oryzae by filter paper disc technique ${ }^{26}$. The concentration of test compounds is $1000 \mu \mathrm{g} / \mathrm{mL}$. After $48 \mathrm{~h}$ treatment, zone of inhibition produced by each compound is measured in $\mathrm{mm}$, as shown in Table 3. Griseofulvin is used as the standard antifungal agent and Dimethyl Formamide as a control.

All the tested compounds show slight to moderate antifungal activity, the data of which is given in Table 3 . 
Table 3. Antifungal activity of the compounds $\mathbf{3}$ and $\mathbf{4 a - h}$

\begin{tabular}{ccccc}
\hline Compound & C. albicans & C. pannical & A. niger & R. oryzae \\
\hline Griseofulvin & +++ & +++ & +++ & +++ \\
$\mathbf{3}$ & + & + & + & + \\
$\mathbf{4 a}$ & ++ & + & ++ & ++ \\
$\mathbf{4 b}$ & ++ & - & ++ & + \\
$\mathbf{4 c}$ & + & + & + & - \\
$\mathbf{4 d}$ & + & - & - & + \\
$\mathbf{4 e}$ & ++ & - & ++ & ++ \\
$\mathbf{4} \mathbf{f}$ & + & - & + & + \\
$\mathbf{4 g}$ & + & - & ++ & + \\
$\mathbf{4 h}$ & ++ & & & + \\
\hline
\end{tabular}

Key to symbols: Inactive $=-($ inhibition zone $<6 \mathrm{~mm})$; slightly active $=+($ inhibition zone 6-9 $\mathrm{mm}$ ); moderately active $=++$ (inhibition zone $9-12 \mathrm{~mm})$; highly active $=+++($ inhibition zone $>12 \mathrm{~mm})$.

\section{Experimental Section}

General Procedures. Melting points are determined in open capillaries on Thomas Hoover apparatus and are uncorrected. ${ }^{1} \mathrm{H}$ NMR and ${ }^{13} \mathrm{C}$ NMR spectra are recorded on Bruker AM 400 instrument (at $400 \mathrm{MHz}$ and $300 \mathrm{MHz}$ respectively) using tetramethylsilane (TMS) as an internal standard and DMSO- $\mathrm{d}_{6}$ as a solvent. Chemical shifts are given in parts per million (ppm). Splitting patterns are designated as follows: s- singlet, d- doublet, t- triplet, q- quartet and mmultiplet. Mass spectra (MS) are recorded on Schimadzu GC-MS. Elemental analysis (C, H, N) is performed on Perkin Elmer 240 analyzer and all compounds are within $\pm 0.4 \%$ of theory unless otherwise specified. All products are purified by recrystallisation. The reactions are followed up and the purity of products is carried out on pre-coated TLC plates (Silica gel $60 \mathrm{~F}_{254}$, Merck), visualizing the spots in ultraviolet light . Column chromatography is performed on Merck silicagel (60-120 mesh). The antimicrobial screening is carried out at Chemo Test Laboratory.

2-Aminobenzothiazole-6-carboxylic acid (1) 2-Aminobenzothiazole-6-carboxylic acid is prepared by a reported method. ${ }^{24}$

2-(2-Chloroacetylamino)benzothiazole-6-carboxylic acid (2). Equimolar solution of 2aminobenzothiazole-6-carboxylic acid 1 ( $0.1 \mathrm{~mole})$ and chloroacetyl chloride $(0.1 \mathrm{~mole})$ in chloroform $(30 \mathrm{~mL})$ in the presence of $\mathrm{K}_{2} \mathrm{CO}_{3}$ is refluxed for about $12 \mathrm{~h}$. Excess of solvent is removed in vacuo and the residue is stirred with water $(50 \mathrm{~mL})$. The residue is washed with $5 \%$ $\mathrm{NaHCO}_{3}$ and subsequently with water. The crude product is dried and crystallized from methanol to furnish pale yellow solid. Yield (81\%); m.p. $160-162{ }^{0} \mathrm{C}$; I.R $\left(\mathrm{KBr}, \mathrm{cm}^{-1}\right) 3430$ 
$(\mathrm{NH}), 1635$ (CONH); ${ }^{1} \mathrm{H}-\mathrm{NMR} \delta 4.29\left(\mathrm{~s}, 2 \mathrm{H}, \mathrm{CH}_{2}\right), 8.0$ (s, 1H, NH), 8.42-8.99 (m, 3H, benzothiazole), 11.1 (s, $1 \mathrm{H}, \mathrm{COOH}$ ); Anal. calcd. for $\mathrm{C}_{10} \mathrm{H}_{7} \mathrm{ClN}_{2} \mathrm{O}_{3} \mathrm{~S}: \mathrm{C} 44.36, \mathrm{H} 2.59, \mathrm{~N} 10.35$, S 11.83 \%. Found: C 44.38, H 2.63, N 10.33, S $11.85 \%$.

2-(2-Imino-4-oxo-thiazolidin-3-yl)benzothiazole-6-carboxylic acid (3). A mixture of 2-(2chloroacetylamino)benzothiazole-6-carboxylic acid 2 (0.01 mole), KSCN (0.02 mole) and dry acetone $(50 \mathrm{~mL})$ is refluxed for about $3 \mathrm{~h}$. Excess of solvent is removed in vacuo and the residue is stirred with water $(50 \mathrm{~mL})$. The solid product is filtered, washed with water and dried. The crude product is crystallized from methanol to furnish light brown solid. Yield (70\%); m.p. 184$186{ }^{\circ} \mathrm{C}$; I.R $\left(\mathrm{KBr}, \mathrm{cm}^{-1}\right) 2980(\mathrm{~N}-\mathrm{H}), 1710(\mathrm{C}=\mathrm{O}), 1570(\mathrm{C}=\mathrm{NH}) ;{ }^{1} \mathrm{H}-\mathrm{NMR} \delta 3.72\left(\mathrm{~s}, 2 \mathrm{H}, \mathrm{CH}_{2}\right)$, $4.83(\mathrm{~s}, 1 \mathrm{H}, \mathrm{C}=\mathrm{NH}), 8.38-8.95(\mathrm{~m}, 3 \mathrm{H}$, benzothiazole), $11.08(\mathrm{~s}, 1 \mathrm{H}, \mathrm{COOH})$; Anal. calcd. for $\mathrm{C}_{11} \mathrm{H}_{7} \mathrm{~N}_{3} \mathrm{O}_{3} \mathrm{~S}_{2}$ : C 45.05, H 2.39, N 14.33, S 21.84 \%. Found: C 45.09, H 2.37, N 14.36, S 21.82 $\%$.

General procedure for the synthesis of 2-[5-(arylidene)-2-imino-4-oxo-thiazolidin-3yl]benzothiazole-6-carboxylic acid (4a-h)

2-(2-Imino-4-oxo-thiazolidin-3-yl)benzothiazole-6-carboxylic acid 3 (0.01 mole) and aromatic aldehyde (0.02 mole) are added to a solution of anhydrous $\mathrm{NaOAc}(0.02$ mole) in $\mathrm{AcOH}$ (30 $\mathrm{mL})$. The mixture is refluxed for $5 \mathrm{~h}$ at $120{ }^{0} \mathrm{C}$ and cooled to room temperature. The solid product is filtered from the mixture, washed with water, dried and crystallized from ethanol.

2-[5-(2-Chlorobenzylidene)-2-imino-4-oxo-thiazolidin-3-yl]benzothiazole-6-carboxylic acid (4a). Yield (61\%); m.p. $255-257{ }^{0} \mathrm{C}$; I.R $\left(\mathrm{KBr}, \mathrm{cm}^{-1}\right) 2970(\mathrm{~N}-\mathrm{H}), 1720(\mathrm{C}=\mathrm{O}), 1550(\mathrm{C}=\mathrm{NH})$; ${ }^{1} \mathrm{H}-\mathrm{NMR} \delta 4.72(\mathrm{~s}, 1 \mathrm{H}, \mathrm{C}=\mathrm{NH})$, 7.06-7.28 (m, 4H, ArH), 7.73 (s, 1H, CH-Ar), 8.36-8.93 (m, 3H, benzothiazole), $11.1(\mathrm{~s}, 1 \mathrm{H}, \mathrm{COOH}) ;{ }^{13} \mathrm{C}$ NMR $\delta 115.7(\mathrm{C}-\mathrm{C}=\mathrm{O}), 121.9,123.7,124.6,126.4$, 126.6 (benzothiazole), 126.8, 127.5, 128.4, 129.5, 131.4, 133.2 (aromatic), 142.3 (CH-aromatic), $151.8(\mathrm{C}=\mathrm{NH}), 154.5,160.3$ (benzothiazole), $166.7(\mathrm{C}=\mathrm{O}), 169.6(\mathrm{COOH})$; Anal. calcd. for $\mathrm{C}_{18} \mathrm{H}_{10} \mathrm{ClN}_{3} \mathrm{O}_{3} \mathrm{~S}_{2}$ : C 51.98, H 2.41, N 10.11, S $15.40 \%$. Found: C 51.95, H 2.45, N 10.15, S $15.43 \%$.

2-[5-(4-Chlorobenzylidene)-2-imino-4-oxo-thiazolidin-3-yl]benzothiazole-6-carboxylic acid (4b). Yield (59\%); m.p. 248-250 ${ }^{0} \mathrm{C}$; I.R (KBr, cm $\left.{ }^{-1}\right) 2964(\mathrm{~N}-\mathrm{H}), 1725(\mathrm{C}=\mathrm{O}), 1560(\mathrm{C}=\mathrm{NH})$; ${ }^{1} \mathrm{H}-\mathrm{NMR} \delta 4.77$ (s, 1H, C=NH), 7.18-7.26 (m, 4H, ArH), 7.44 (s, 1H, CH-Ar), 8.42-8.99 (m, 3H, benzothiazole), $11.16(\mathrm{~s}, 1 \mathrm{H}, \mathrm{COOH})$; Anal. calcd. for $\mathrm{C}_{18} \mathrm{H}_{10} \mathrm{ClN}_{3} \mathrm{O}_{3} \mathrm{~S}_{2}: \mathrm{C} 51.98, \mathrm{H} \mathrm{2.41,} \mathrm{N}$ 10.11, S 15.40 \%. Found: C 51.93, H 2.43, N 10.14, S $15.42 \%$.

2-[5-(4-Hydroxybenzylidene)-2-imino-4-oxo-thiazolidin-3-yl]benzothiazole-6-carboxylic

acid (4c). Yield (69\%); m.p. 244-246 ${ }^{0} \mathrm{C}$; I.R (KBr, cm $\left.{ }^{-1}\right) 3580(\mathrm{ArOH}), 2940$ (N-H), 1732 $(\mathrm{C}=\mathrm{O}), 1575(\mathrm{C}=\mathrm{NH})$; ${ }^{1} \mathrm{H}-\mathrm{NMR} \delta 4.69$ (s, 1H, C=NH), 5.06 (s, 1H, Ar-OH), 6.64-7.17 (m, 4H, ArH), 7.47 (s, 1H, CH-Ar), 8.41-8.98 (m, 3H, benzothiazole), 11.04 (s, 1H, COOH); Anal. calcd. for $\mathrm{C}_{18} \mathrm{H}_{11} \mathrm{~N}_{3} \mathrm{O}_{4} \mathrm{~S}_{2}$ : C 54.41, H 2.77, N 10.58, S $16.12 \%$. Found: C 54.47, H 2.79, N 10.52, S $16.07 \%$. 
2-[5-(3-Bromobenzylidene)-2-imino-4-oxo-thiazolidin-3-yl]benzothiazole-6-carboxylic acid (4d). Yield (58\%); m.p. 278-280 ${ }^{0} \mathrm{C}$; I.R (KBr, cm $\left.{ }^{-1}\right) 2955(\mathrm{~N}-\mathrm{H}), 1715(\mathrm{C}=\mathrm{O}), 1565(\mathrm{C}=\mathrm{NH})$; ${ }^{1} \mathrm{H}-\mathrm{NMR} \delta 4.65$ (s, 1H, C=NH), 7.08-7.45 (m, 4H, ArH), 7.51 (s, 1H, CH-Ar), 8.39-8.96 (m, 3H, benzothiazole), 11.2 (s, $1 \mathrm{H}, \mathrm{COOH}$ ); Anal. calcd. for $\mathrm{C}_{18} \mathrm{H}_{10} \mathrm{BrN}_{3} \mathrm{O}_{3} \mathrm{~S}_{2}$ : C 46.96, $\mathrm{H}$ 2.17, N 9.13, S $13.91 \%$. Found: C 46.91, H 2.14, N 9.10, S $13.96 \%$.

2-[2-Imino-5-(4-methoxybenzylidene)-4-oxo-thiazolidin-3-yl]benzothiazole-6-carboxylic acid (4e). Yield (55\%); m.p. 251-253 ${ }^{0} \mathrm{C}$; I.R (KBr, cm $\left.{ }^{-1}\right) 2960(\mathrm{~N}-\mathrm{H}), 1740(\mathrm{C}=\mathrm{O}), 1558$ $(\mathrm{C}=\mathrm{NH}) ;{ }^{1} \mathrm{H}-\mathrm{NMR} \delta 3.71\left(\mathrm{~s}, 3 \mathrm{H}, \mathrm{Ar}-\mathrm{OCH}_{3}\right), 4.70(\mathrm{~s}, 1 \mathrm{H}, \mathrm{C}=\mathrm{NH}), 6.74-7.17$ (m, 4H, ArH), 7.46 (s, 1H, CH-Ar), 8.44-8.97 (m, 3H, benzothiazole), $11.12(\mathrm{~s}, 1 \mathrm{H}, \mathrm{COOH})$; Anal. calcd. for $\mathrm{C}_{19} \mathrm{H}_{13} \mathrm{~N}_{3} \mathrm{O}_{4} \mathrm{~S}_{2}$ : C 55.47, H 3.16, N 10.22, S 15.57 \%. Found: C 55.51, H 3.13, N 10.27, S 15.54 $\%$.

2-[2-Imino-5-(3-nitrobenzylidene)-4-oxo-thiazolidin-3-yl]benzothiazole-6-carboxylic acid (4f). Yield (65\%); m.p. 266-268 ${ }^{0} \mathrm{C}$; I.R (KBr, cm $\left.{ }^{-1}\right) 2972(\mathrm{~N}-\mathrm{H}), 1705(\mathrm{C}=\mathrm{O}), 1547(\mathrm{C}=\mathrm{NH})$, 1348,1524 $\left(\mathrm{NO}_{2}\right) ;{ }^{1} \mathrm{H}-\mathrm{NMR} \delta 4.79$ (s, 1H, C=NH), 7.43 (s, 1H, CH-Ar), 7.57-8.33 (m, 4H, ArH), 8.40-8.97 (m, 3H, benzothiazole), $11.09(\mathrm{~s}, 1 \mathrm{H}, \mathrm{COOH})$; Anal. calcd. for $\mathrm{C}_{18} \mathrm{H}_{10} \mathrm{~N}_{4} \mathrm{O}_{5} \mathrm{~S}_{2}$ : C 50.70, H 2.35, N 13.15, S $15.02 \%$. Found: C 50.73, H 2.31, N 13.12, S $15.07 \%$.

2-[2-Imino-5-(4-nitrobenzylidene)-4-oxo-thiazolidin-3-yl]benzothiazole-6-carboxylic acid (4g). Yield (68\%); m.p. 273-275 ${ }^{0}$ C; I.R (KBr, cm $\left.{ }^{-1}\right) 2968(\mathrm{~N}-\mathrm{H}), 1700(\mathrm{C}=\mathrm{O}), 1552(\mathrm{C}=\mathrm{NH})$, 1355,1529 $\left(\mathrm{NO}_{2}\right) ;{ }^{1} \mathrm{H}-\mathrm{NMR} \delta 4.76(\mathrm{~s}, 1 \mathrm{H}, \mathrm{C}=\mathrm{NH}), 7.48$ (s, 1H, CH-Ar), 7.62-8.18 (m, 4H, ArH), 8.34-8.90 (m, 3H, benzothiazole), $11.06(\mathrm{~s}, 1 \mathrm{H}, \mathrm{COOH})$; Anal. calcd. for $\mathrm{C}_{18} \mathrm{H}_{10} \mathrm{~N}_{4} \mathrm{O}_{5} \mathrm{~S}_{2}$ : C 50.70, H 2.35, N 13.15, S $15.02 \%$. Found: C 50.67, H 2.39, N 13.13, S $15.01 \%$.

2-(5-Benzylidene-2-imino-4-oxo-thiazolidin-3-yl)benzothiazole-6-carboxylic acid (4h). Yield (63\%); m.p. 238-240 ${ }^{0} \mathrm{C}$; I.R (KBr, $\left.\mathrm{cm}^{-1}\right) 2973(\mathrm{~N}-\mathrm{H}), 1735(\mathrm{C}=\mathrm{O}), 1545(\mathrm{C}=\mathrm{NH}) ;{ }^{1} \mathrm{H}-\mathrm{NMR} \delta$ $4.63(\mathrm{~s}, 1 \mathrm{H}, \mathrm{C}=\mathrm{NH}), 7.12-7.28(\mathrm{~m}, 5 \mathrm{H}, \mathrm{ArH}), 7.42(\mathrm{~s}, 1 \mathrm{H}, \mathrm{CH}-\mathrm{Ar}), 8.44-9.00(\mathrm{~m}, 3 \mathrm{H}$, benzothiazole), 11.02 (s, $1 \mathrm{H}, \mathrm{COOH})$; Anal. calcd. for $\mathrm{C}_{18} \mathrm{H}_{11} \mathrm{~N}_{3} \mathrm{O}_{3} \mathrm{~S}_{2}$ : C 56.69, H 2.88, N 11.02, S 16.80 \%. Found: C 56.71, H 2.83, N 11.05, S $16.83 \%$.

\section{Acknowledgements}

The authors are thankful to Dr. Atul Pusalkar and Dr. Borna Basu, Chemo Test Laboratory for antimicrobial screening of the compounds. The authors are also grateful to the U.G.C. for the financial assistance. 


\section{References}

1. (a) Vigorita, M. G.; Ottana, R.; Monforte, F.; Maccari, R.; Trovato, A.; Monforte, M. T.; Taviano, M. F. Bioorg. Med. Chem. Lett. 2001, 11, 2791. (b) Chande, M. S.; Suryanarayan, V. J. Chem. Res. 2005, 6, 345. (c) Kavitha, C. V.; Basappa, S.; Swamy, N.; Mantelingu, K.; Doreswamy, S.; Sridhar, M. A.; Prasad, S.; Rangappa, K. S. Bioorg. Med. Chem. 2006, 14, 2290. (d) Shiradkar, M.; Shivaprasad, H. N. Asian J. Chem. 2006, $18,331$.

2. Diurno, M. V.; Mazzoni, O.; Izzo, A. A.; Bolognese, A. Il Farmaco 1997, 52, 237.

3. Ergene, N.; Capan, G. Il Farmaco 1994, 49, 449.

4. Desai, S. B.; Desai, P. B.; Desai, K. R. Asian J. Chem. 1999, 2, 363; Sharma, R. C.; Kumar, D. J. Indian Chem. Soc. 2000, 77, 492; Piscapo, E.; Diurno, M. V.; Gagliardi, R.; Mazzoni, O. Boll. Soc. Ital. Biol. Sper. 1989, 65, 853.

5. Ueno, H.; Oe, T.; Snehiro, I.; Nakamura, S. US Patent 5594116, 1997; Chem. Abstr. 1977, $126,157507 \mathrm{p}$.

6. Previtera, T.; Vigorita, M. G.; Bisila, M.; Orsini, F.; Benetolla, F.; Bombieri, G. Eur. J. Med. Chem. 1994, 29, 317; Diurno, M. V.; Mazzoni, O.; Correale, G.; Monterry, I. G.. Il Farmaco 1999, 54, 579.

7. Ebeid, M. Y.; Fathallah, O. A.; El-Zaher, M. I.; Kamel, M. M.; Abdon, W. A.; Anwar, M. M. Bull. Fac. Pharm. 1996, 34, 125.

8. Rawal, R. K.; Prabhakar, Y. S.; Katti, S. B.; De Clercq, E. Bioorg. Med. Chem. 2005, 13, 6771.

9. Kato, T.; Ozaki, T.; Tamura, K. J. Med. Chem. 1999, 42, 3134; Hara, A.; Suzuki, T.; Hashizume, H.; Shishido, N.; Nakamura, M.; Ushikube, F.; Abiko, Y. Eur. J. Pharmacol. 1999, 385, 81.

10. Tanabe, Y.; Suzukamo, G.; Komuro, Y.; Imanishi, N.; Morooka, S.; Enomoto, M.; Kojima, A.; Sanemitsu, Y.; Mizutani, M. Tetrahedron Lett. 1991, 32, 379.

11. Kato, T.; Ozaki, T.; Ohi, N. Tetrahedron: Asymmetry 1999, 10, 3963.

12. Adachi, Y.; Suzuki, Y.; Homma, N.; Fukazawa, M.; Tamura, K.; Nishie, I.; Kuromaru, O. Eur. J. Pharmacol. 1999, 367, 267.

13. Ottana, R.; Mazzon, E.; Dugo, L.; Monforte, F.; Maccari, R.; Sautebin, L.; De Luca, G.; Vigorita, M. G.; Alcaro, S.; Ortuso, F. Eur. J. Pharmacol. 2002, 448, 71.

14. Koike, H.; Imanashi, N.; Natsume, Y.; Morooka, S. Eur. J. Pharm. Mol. Pharm. 1994, 269; Tanabe, Y.; Yamamoto, H.; Murakami, M.; Yanagi, K.; Kubota, Y.; Okumura, H.; Sanemitsu, Y.; Suzukamo, G. J. Chem. Soc. Perkin Trans. I 1995, 7, 935; Tanabe, Y.; Komuro, Y.; Imanishi, N.; Morooka, S.; Enomoto, M.; Kojima, A.; Sanemitsu, Y.; Mizutani, M. Tetrahedron. Lett. 1991, 32, 379.

15. Kato, Y.; Kita, Y.; Nishio, M.; Hirasawa, Y.; Ito, K.; Yamanaka, T.; Motoyama, Y.; Seki, J. Eur. J. Pharmacol. 1999, 384, 197.

16. Voss, M. E.; Carter, P. H.; Tebben, A. J.; Scherle, P. A.; Brown, G. D.; Thompson, L. A.; Xu, M.; Lo, Y. C.; Yang-Liu, R. R. Q. Bioorg. Med. Chem. Lett. 2003, 13, 533. 
17. Lakhan, R.; Singh, O. P. J. Ind. Chem. Soc. 1984, 61, 784.

18. Bhargava, P. N.; Prakash, S.; Lakhan, R. Ind. J. Chem. 1981, $20 B, 927$.

19. Lakhan, R. Agric. Biol. Chem. 1982, 46, 557.

20. Russo, F.; Romeo, G.; Santagati, N. A.; Caruso, A.; Cutuli, V.; Amore, D. Eur. J. Med. Chem. 1994, 29, 569.

21. Baltork, I. M.; Khosropour, A. R.; Hojati, S. F. Monatshefte fur Chemie / Chemical Monthly 2007, 138, 663 .

22. Katsura, Y.; Inoue, Y.; Nishino, S.; Tomoi, M.; Takasugi, H. Chem. Pharm. Bull (Tokyo) 1992, 40, 1818.

23. Kuhler, T. C.; Swanson, M.; Shcherbuchin, V.; Larsson, H.; Mellgard, B.; Sjostrom, J. E. J. Med. Chem. 1998, 41, 1777.

24. Nikolyukin, Y. A.; Gibboni, D. J. U.S. 5710012, 1998.

25. British Pharmacopoeia. 2005, Vol. IV, p. A300 Appendix XIV.

26. Vincent, J. G.; Vincent, H. W. Proc. Soc. Exptl. Biol. Med. 1944, 55, 162. 